\title{
Neutron capture cross sections: from theory to experiments and back
}

\author{
A. Mengoni ${ }^{1}$ \\ CERN, CH-1211 Geneva 23, Switzerland, and \\ ENEA, Via Don Fiammelli, 2 - 40129 Bologna, Italy
}

\begin{abstract}
The method for an experimental determination of the stellar enhancement factor for the cross section of the ${ }^{151} \mathrm{Sm}(n, \gamma)$ reaction process is proposed. This study offered the pretext for an excursus on the interconnections between capture and dissociation reactions and the interplay between theory and experiments in the determination of neutron capture cross sections.
\end{abstract}

\section{INTRODUCTION}

One of the most difficult issues for the accurate determination of stellar reaction rates is the evaluation of the so-called stellar enhancement factor (SEF). This factor simply relates the cross section measured in the laboratory to the cross section in a stellar environment, $\sigma^{*}$, by $\sigma^{*} \equiv \mathrm{SEF} \times \sigma^{l a b}$. In capture reactions, this means that for the determination of the SEF, an evaluation of the capture cross section for nuclei in excited states needs to be done. In same cases the SEF is close to unity and the effect on the stellar cross section is marginal. However, there are several important cases in which excited states of the target nuclei are strongly populated in a stellar environment. There, an accurate determination of the SEF is mandatory. Just to mention one example in which this is the case one can consider the SEF of the $\sigma_{n, \gamma}$ of the ${ }^{186}$ Os and ${ }^{187}$ Os. The latter, in particular, has an excited state at only $9.8 \mathrm{KeV}$ excitation energy and at stellar temperature of the order of $k T \approx 30 \mathrm{KeV}$ can be populated up to $50 \%$. This implies a $20 \%$ difference in the stellar Maxwellian averaged capture cross section ratio of ${ }^{186}$ Os to ${ }^{187}$ Os. In turn, if one uses the Re/Os clock for the age determination of the galactic nucleosynthesis, one obtains an increase of $2 \mathrm{Gyr}$ in the age. It is therefore necessary to obtain an accurate determination of the SEF. This is usually done in model calculations based on the Hauser-Feshbach statistical model theory (HFSM). Measurements of the capture cross sections for target nuclei in their ground-state allow for checking the accuracy of the model calculations. However, an experimental determination of the SEF would strongly enhance the reliabil- ity of the evaluated reaction rates in stellar environment. The possibility to measure directly the cross section from nuclei in excited states is scarce if not completely null, because nuclei in excited states (which are not isomers) live a marginal fraction of seconds. It is proposed here to use the inverse reaction channel of the neutron capture process to deduce the SEF for one important neutron capture cross section: the ${ }^{151} \mathrm{Sm}(n, \gamma)$. This reaction cross section has been measured recently at the CERN n_TOF facility [1] as well as at the Forschungszentrum, Karlsruhe [2]. These measurements provide confidence on the experimental determination of the Maxwellian averaged cross section (MACS) for the ground-state capture. On the other hand, the SEF for this reaction needs to be derived by theoretical calculation which may produce different results. The possibility of an experimental determination of the SEF seems therefore appealing.

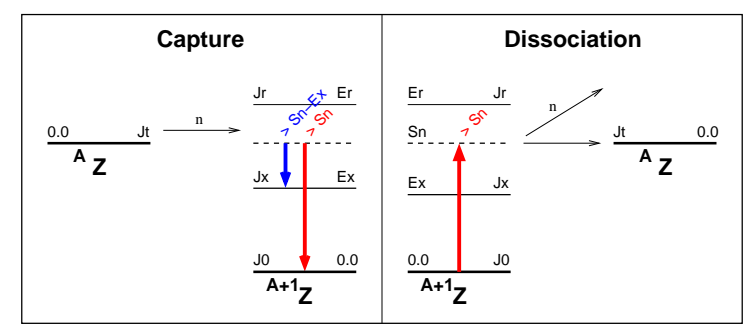

FIGURE 1. Schematic view of the neutron capture and dissociation processes

\footnotetext{
1 e-mail: alberto.mengoni@cern.ch
} 


\section{DIRECT AND INVERSE REACTION CHANNELS}

The time-reversal invariance of nuclear reactions which leads to the detailed-balance relation between reactions in the "direct" and "inverse" channels has been widely used for deriving reaction cross sections which can be accessed in the laboratory only in one of the time directions. A schematic view of the capture reaction process and its time-reversals invariant is shown in Figure 1 The relation between the cross sections in the case of a neutron capture reaction process, $n+{ }^{A} \mathrm{Z} \rightarrow{ }^{A+1} \mathrm{Z}+\gamma$, is simply given by

$$
\sigma_{n, \gamma}=\frac{k_{\gamma}^{2}}{k_{n}^{2}} \frac{2 J_{A+1}+1}{2 J_{A}+1} \sigma_{\gamma, n}
$$

where $k_{n}$ is the incident neutron wave number and $k_{\gamma}=$ $\varepsilon_{\gamma} / \hbar c$ is the $\gamma$-ray wave number related to the $\gamma$-ray transition energy $\varepsilon_{\gamma}$. The dissociation can be induced by real photons, or by the virtual photon field generated by a high- $Z$ target as in the Coulomb dissociation process. The latter reaction process is nowadays widely used in experiments with radioactive ion beams, in which also the reaction kinematics is inverted (see for example [3]). One important difference to notice between the two reaction processes is that, while in the capture channel, all the states in the $n+{ }^{A} \mathrm{Z}$ composite system are populated, in the inverse dissociation process only the ground-state of the composite system is involved. Therefore, the timereversal invariance is fully symmetrical only for groundstate transitions. It is also important to note here that excited states in the residual nucleus can be populated by the dissociation process. As will be shown in detail below, the proposal of the present work is based on this consideration.

On the experimental side, $(\gamma, n)$ reactions have been used recently for the determination of some capture reaction rates of unstable isotopes of branching points in the $s$ process path, with two different techniques. An activation method based on a bremsstrahlung photon spectrum such as the one of the S-DALINAC at Darmstadt Technical University. In this case, a photon beam with different bremsstrahlung end-point energies is used to activate a target material whose decay is detected off-line after the irradiation. A second method is based on the use of laser inverse-Compton scattering gamma-ray sources. In this case, a monochromatic gamma-ray beam is used to induce prompt photo-neutron (or photo- $p$, photo- $\alpha$, etc.) emission in the dissociation process. Both techniques are described in a recent review paper [4] in which details of the methods used to derive the capture reaction rate from $(\gamma, n)$ measurements are given. For both methods, a model calculation of the $(\gamma, n)$ cross section is required. The calculated cross section is then normalized to the experimental data obtained for the ground-state transitions and then the same normalization factor is used in the evaluation of the cross section for transitions to the other excited states.

\section{SEF: FROM THEORY TO EXPERIMENT}

The ${ }^{151} \mathrm{Sm}(n, \gamma){ }^{152} \mathrm{Sm}$ reaction has important implications in nuclear astrophysics as it can be used to constrain the temperature conditions of the He burning phase of AGB stars. With its 93 years $\beta$-decay half-life, ${ }^{151} \mathrm{Sm}$ represents a branching point in the $s$ process path which is influencing the isotopic abundance ratio of the two $s$ only $\mathrm{Gd}$ isotopes ${ }^{152} \mathrm{Gd}$ and ${ }^{154} \mathrm{Gd}$. Since the $\beta$-decay half-life of ${ }^{151} \mathrm{Sm}$ is temperature dependent, once that its capture cross section is determined, a link between the temperature conditions and the isotopic abundance ratio between the two $\mathrm{Gd}$ isotopes can be established.

The ${ }^{151} \operatorname{Sm}(n, \gamma){ }^{152} \mathrm{Sm}$ cross section has been recently measured at the CERN n_TOF facility [1]. The result for the MACS-30 (MACS at $k T=30 \mathrm{keV}$ ) is $3100 \pm 160$ $\mathrm{mb}$. This result has been confirmed in a measurement performed at FZK [2] in which the MACS-30 result obtained is $3031 \pm 68 \mathrm{mb}$. Theoretical estimates of the capture cross section based on HF statistical model theory produces much lower values, typically around $2000 \mathrm{mb}$. In any case, the energy dependence of the cross section is well reproduced by the model calculation and, once that the MACS value is obtained in the laboratory, the calculated cross section can be normalized.

${ }^{151} \mathrm{Sm}$ has several low-lying levels which can be populated under stellar conditions. For example, the first excited state at $4.8 \mathrm{keV}$ is populated at a $30 \%$ level at temperatures of $k T=30 \mathrm{KeV}$. The determination of the $\mathrm{SEF}$ for the ${ }^{151} \mathrm{Sm}(n, \gamma){ }^{152} \mathrm{Sm}$ reaction is therefore important for a reliable application to AGB star modeling. A schematic view of the situation in reported in Figure 2

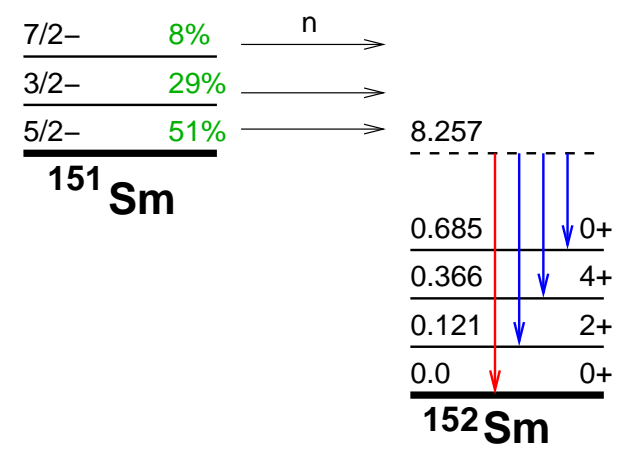

FIGURE 2. Schematic view of the neutron capture by ${ }^{151} \mathrm{Sm}$. The population probabilities of the first three excitated target states are indicated for a temperature of $k T=30 \mathrm{KeV}$. 
As mentioned in the introduction, the SEF can be calculated. This is normally done with the use of the HauserFeshbach statistical model theory. The main ingredients in these calculations are the optical model parameters representing the interaction of the neutron with the target, furnishing the neutron transmission coefficients for the neutron-nucleus interaction. In addition, the representation of the density of nuclear states at excitation energies up to the neutron separation threshold and up to $\approx 1 \mathrm{MeV}$ above. Finally, the electric dipole resonance parameters are used to evaluate the $\gamma$-ray strength functions.

The NON-SMOKER calculation of the SEF for the ${ }^{151} \mathrm{Sm}(n, \gamma){ }^{152} \mathrm{Sm}$ reaction is 0.87 at $k T=30 \mathrm{KeV}$ [5], while in the calculation performed for the present work we have obtained 0.93 . This discrepancy is most likely due to different parameterizations of the HauserFeshbach theory which are used in model calculations. Therefore, the accuracy of the SEF calculation cannot guaranteed by the HFSM theory itself.

\section{THE ${ }^{152} \mathbf{S m}(\gamma, n){ }^{151} \mathbf{S m}$ CASE}

The ${ }^{152} \mathrm{Sm}(\gamma, n){ }^{151} \mathrm{Sm}$ reaction can be measured with both the techniques mentioned above. There is, however, a peculiarity in this reaction. In fact, starting from the $J^{\pi}=0^{+}$ground-state of ${ }^{152} \mathrm{Sm}$, only excited states in ${ }^{151} \mathrm{Sm}$ can be populated by the strongest E1 transitions and with $s$-wave neutrons in the continuum. In particular, the $J^{\pi}=5 / 2^{-}$can only be populated with $d$-wave neutrons left in the continuum. The $J^{\pi}=3 / 2^{-}$first excited state at $E_{x}=4.82 \mathrm{KeV}$ in ${ }^{151} \mathrm{Sm}$ is populated from the neutron emission threshold and $s$-wave neutrons in the continuum. The next possible excitation is the 5 th excited state at $E_{x}=104.8 \mathrm{KeV}$, also a $J^{\pi}=3 / 2^{-}$state. This situation is schematically shown in Figure 3

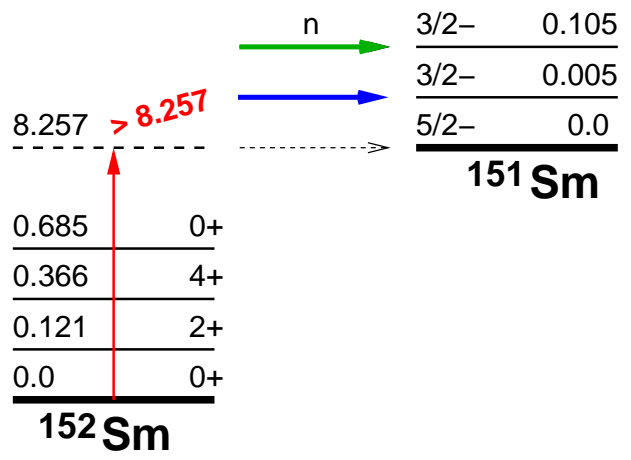

FIGURE 3. Dissociation of ${ }^{152} \mathrm{Sm}$ into ${ }^{151} \mathrm{Sm}+n$.

The HFSM results of the calculation of the $(\gamma, n)$ reaction process are shown in Figure 4 There, the cross sections $\sigma_{\gamma, n}$ for the various reaction channels are shown in the energy region above the neutron emission thresh- old. As expected, the only open channel up to $\approx 100$ $\mathrm{KeV}$ above threshold is the dissociation leading to the ${ }^{151} \operatorname{Sm}\left(J^{\pi}=3 / 2^{-}\right)$state. Therefore, in an actual experiment, this would produce the information needed to derive the capture cross section of ${ }^{151} \mathrm{Sm}$ from its first excited state.

As the excitation energy increases, the second dissociation channel at $E_{x}=104.8 \mathrm{KeV}$ opens up. Then, a measurement of the $(\gamma, n)$ cross section above this energy range would include the effect of this state and in turn, allow for an experimental determination of the timereversal neutron capture process from this state. Only at higher excitation energies, the effect of the ground-state starts to contribute, but only to a few percent level.

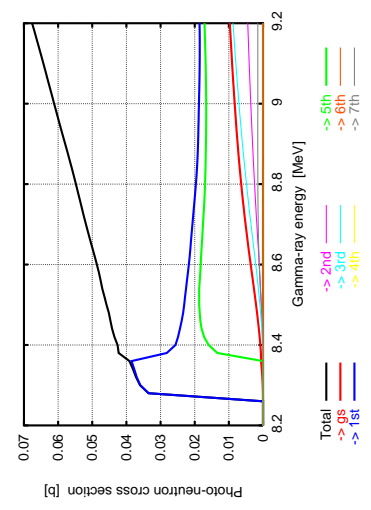

FIGURE 4. HFSM calculation of the ${ }^{152} \mathrm{Sm}(\gamma, n){ }^{151} \mathrm{Sm}$ cross section for dissociation into the different ${ }^{151} \mathrm{Sm}+n$ channels.

The HFSM calculations have been performed using the nuclear level density parametrization of reference [6]. The parameters have been fixed to reproduce the experimental level spacings at the neutron separation energies which, in the present case, are available for both the isotopes involved [7]. The Moldauer [8] optical model parameters have been used for the neutron transmission function calculations and experimental parameters of the Giant Dipole Resonance [9] for the calculation of the $\gamma$-ray strength function. The cross section has been renormalized in order to reproduce the measured ${ }^{151} \mathrm{Sm}(n, \gamma){ }^{152} \mathrm{Sm}$ MACS-30. This normalization factor turned out to be 1.7 for the present HFSM calculations as well as for the NON-SMOKER result [5].

The absolute value of the dissociation cross section is of the same order as that of measurements already performed (see for example the report on the ${ }^{186} \mathrm{~W}(\gamma, n)$ measurement [10]), making the proposal for an actual experiment quite realistic. 


\section{CONCLUSION}

The experimental determination of the SEF for the ${ }^{151} \mathrm{Sm}(n, \gamma)$ could be possible by a measurement of the time-reversal invariant reaction process, the ${ }^{152} \mathrm{Sm}(\gamma, n){ }^{151} \mathrm{Sm}$ reaction. This reaction can only populate excited states in the ${ }^{151} \mathrm{Sm}+n$ residual channel for excitation energies just above threshold. The technique has been used for the determination of capture cross sections of unstable nuclei at $s$ process branching points and could, in this specific case lead to the experimental determination of a SEF for the first time.

\section{ACKNOWLEDGMENTS}

I would like to thank Franz Käppeler for several fruitful discussions on the subject of the present work.

\section{REFERENCES}

1. U. Abbondanno et al. (The $\mathrm{n}_{-} \mathrm{TOF}$ Collaboration), Phys. Rev. Letters 93 (2004) 161103.

2. K. Wisshak, et al., Forschungszentrum Karlsruhe Report FZKA 6996 (2004).

3. N. Fukuda, T. Nakamura, N. Aoi, N. Imai, M. Ishihara, T. Kobayashi, H. Iwasaki, T. Kubo, A. Mengoni, M. Notani, H. Otsu, H. Sakurai, S. Shimoura, T. Teranishi, Y.X. Watanabe, and K. Yoneda, Phys. Rev. C 70 (2004) 054606.

4. H. Utsunomiya, P. Mohr, A. Zilges, and M. Rayet, Nucl. Phys. A, (2004) in press.

5. T. Rauscher, and F.-K. Thielemann, Atomic Data Nuclear Data Tables 79 (2001) 47.

6. A. Mengoni, and Y. Nakajima, The Journal of Nuclear Science and Technology 31 (1994) 151

7. S. F. Mughabghab, M. Divadeenam, and N. E. Holden, Neutron Cross-sections, Vol. I (Academic Press, 1981).

8. P.A. Moldauer, Nucl. Phys. 47 (1963) 65.

9. P. Carlos, H. Beil, R. Bergere, A. Lepretre, A. De Miniac, A. Veyssiere, Nucl. Phys. A225 (1974) 171.

10. K. Sonnabend, P. Mohr, K. Vogt, A. Zilges, A. Mengoni, T. Rauscher, H. Beer, F. Käppeler, and R. Gallino, ApJ 583 (2003) 506. 